\title{
Repeated oral ketamine for out-patient treatment of resistant depression: randomised, double-blind, placebo-controlled, proof-of-concept study
}

Yoav Domany*, Maya Bleich-Cohen* , Ricardo Tarrasch, Roi Meidan, Olga Litvak-Lazar, Nadav Stoppleman, Shaul Schreiber, Miki Bloch, Talma Hendler and Haggai Sharon

\section{Background}

Ketamine has been demonstrated to improve depressive symptoms.

\section{Aims}

Evaluation of efficacy, safety and feasibility of repeated oral ketamine for out-patients with treatment-resistant depression (TRD)

\section{Method}

In a randomised, double-blind, placebo-controlled, proof-ofconcept trial, 41 participants received either $1 \mathrm{mg} / \mathrm{kg}$ oral ketamine or placebo thrice weekly for 21 days (ClinicalTrials.gov Identifier: NCT02037503). Evaluation was performed at baseline, 40 and 240 min post administration and on days 3, 7, 14 and 21. The main outcome measure was change in Montgomery-Åsberg Depression Rating Scale (MADRS).

\section{Results}

Twenty-two participants were randomised to the ketamine group, and 19 to the control, with $82.5 \%(n=33)$ completing the study. In the ketamine group, a decrease in depressive symptoms was evident at all time points, whereas in the control group a decrease was evident only 40 min post administration.
The reduction in MADRS score on day 21 was 12.75 in the ketamine group versus 2.49 points with placebo $(P<0.001)$. Six participants in the ketamine group (27.3\%) achieved remission compared with none of the controls $(P<0.05)$. The number needed to treat for remission was 3.7. Side-effects were mild and transient.

\section{Conclusions}

Repeated oral ketamine produced rapid and persistent amelioration of depressive symptoms in out-patients with TRD, and was well tolerated. These results suggest that add-on oral ketamine may hold significant promise in the care of patients suffering from TRD in the community.

\section{Declaration of interest}

None.

\section{Keywords}

Depressive disorders; antidepressants; ketamine; treatment resistant depression; randomized controlled trial.

\section{Copyright and usage}

(c) The Royal College of Psychiatrists 2018
Current treatment strategies for depression fail to achieve remission in about $30 \%$ of patients with depression, ${ }^{1}$ which is often considered to be treatment-resistant depression (TRD). ${ }^{2}$ Intravenous ketamine, a glutamate modulator, has been demonstrated to act as a novel antidepressant ${ }^{3,4}$ with an extended effect following repeated infusions ${ }^{5}$ while maintaining a good safety profile. ${ }^{4,5}$ However, intravenous administration presents major obstacles to clinical applicability, especially in a community setting. Several other routes of administration have been explored such as intranasal, ${ }^{6}$ sublingual ${ }^{7}$ and subcutaneous. ${ }^{8}$ All these were preliminary studies or case series, and reported good safety and efficacy. Oral administration of ketamine, however, has scarcely been reported for depression whereas in chronic pain management ${ }^{9}$ oral dosing with ketamine is a recognised route of administration, even on a prolonged basis in the community. Two studies have evaluated oral ketamine for depression in specific medial conditions, such as hospice patients $^{10}$ and those with comorbid chronic pain. ${ }^{11}$ Both showed efficacy with good safety and tolerability. Oral ketamine for TRD was only described in a single case report ${ }^{12}$ and recently in a retrospective case series, ${ }^{13}$ though the latter concluded that oral ketamine is only modestly effective. A need for quality trials assessing repeated oral administration of ketamine for longer periods in the community has been emphasised. ${ }^{14}$ The aim of this study was to evaluate the effect of add-on, repeated

* These authors contributed equally to the work. oral administration of ketamine on depression severity in patients with TRD, and assess its safety and feasibility.

\section{Method}

\section{Participants}

The study was conducted at Tel Aviv Sourasky Medical Center between May 2014 and July 2016. Inclusion criteria were age 18-75 years, a diagnosis of major depressive disorder, as determined by the Mini International Neuropsychiatric interview (M.I.N.I.) ${ }^{15}$ and a score of 19 or greater on the Montgomery-Åsberg Depression Rating Scale (MADRS). ${ }^{16}$ The definition of TRD (in the current episode) that we used was an inadequate response to documented therapeutic trials of at least two antidepressants of an adequate dose and duration. ${ }^{2}$ We documented the current pharmacological treatment and any other treatments in the current depressive episode. A senior psychiatrist carried out all screening assessments. Exclusion criteria included psychotic disorder or psychotic symptoms, bipolar disorder, alcohol or substance misuse, unstable medical illness or contraindications to ketamine administration.

The study was approved by the Institutional Review Boards of the Tel Aviv Sourasky Medical Center. The trial is registered at http://clinicaltrials.gov (NCT02037503). All participants provided written informed consent after receiving a full explanation regarding the study protocol. All participants were Hebrew speakers with adequate language comprehension. Recruitment for the study ended in July 2016, when we reached our predetermined recruitment goal. 


\section{Study design}

A double-blind, randomised, placebo-controlled proof-of-concept trial was conducted for 21 days with an additional follow-up session at day 28 (Fig. 1). Participants were out-patients referred from the community or the hospital's ambulatory service. Following baseline assessments, participants were randomly assigned to either the active treatment or the inactive placebo group (normal saline). The study intervention was given as addon therapy to the patient's usual prescribed care (treatment as usual). Participants were prescribed a stable treatment regimen for at least 1 month prior to study enrolment and were instructed to continue their usual treatment for the whole trial duration.

\section{Assessment}

Participants were assessed for study eligibility in a personal interview conducted by a senior psychiatrist, within $24 \mathrm{~h}$ prior to first drug administration. The primary outcome measure was change in depression severity, as determined by the MADRS scores. Secondary outcomes were achievement of clinical remission, defined as a MADRS score $\leq 10$ points, ${ }^{17}$ and response to treatment, defined as a reduction of more than $50 \%$ of the baseline depression score, both at day 21 .

\section{Depression severity}

The MADRS is a depression scale, widely used in research, particularly in settings where change in depressive symptoms should be captured, ${ }^{16}$ including ketamine trials. ${ }^{5,18}$ The MADRS normally reflects the past 7 days; we used a revised MADRS for the period since last evaluation. MADRS baseline scores were documented up to 1 day before drug administration by a senior psychiatrist and then again at the first and last day of the trial. Additional, repeated assessments were carried out by trained researches. All raters were masked to participants' allocations.

\section{Adverse effects and serious adverse events}

Data was collected with direct patient questioning at every meeting. During the first administration, physiological measurements of blood pressure, pulse rate and arterial oxygen saturation were performed. All adverse events were documented in the participant's chart.

\section{Dose and drug administration}

The active drug was a solution of racemic ketamine $50 \mathrm{mg} / 1 \mathrm{~mL}$. Evidence for determining optimal dosing and regimen are scarce and oral administration of ketamine has a low, unstable, bioavailability, which might be affected by food intake and personal metabolic profile. ${ }^{9}$ We chose to extrapolate the dosage from those described for intravenous administration $(0.5 \mathrm{mg} / \mathrm{kg})$ and tailor it based on expected oral absorption $(17-23 \%)^{9}$ and specific participants' body weight. This is in accordance with previously reported dose regimes of oral ketamine for depression. ${ }^{11,13}$ Therefore, we decided to administer $1 \mathrm{mg} / \mathrm{kg}$. Regarding the dosing schedule, because twice and thrice weekly administration has been suggested to have similar results, ${ }^{5}$ we chose to give it thrice weekly considering the lower dose given, and the unstable oral bioavailability.

Participants meeting inclusion criteria were randomised (1:1) to oral ketamine or matched oral placebo. Randomisation was performed by a non-rater physician who was in charge of safety and was the only team member who was not masked. In order to randomly allocate patients, he predetermined the order of inclusion

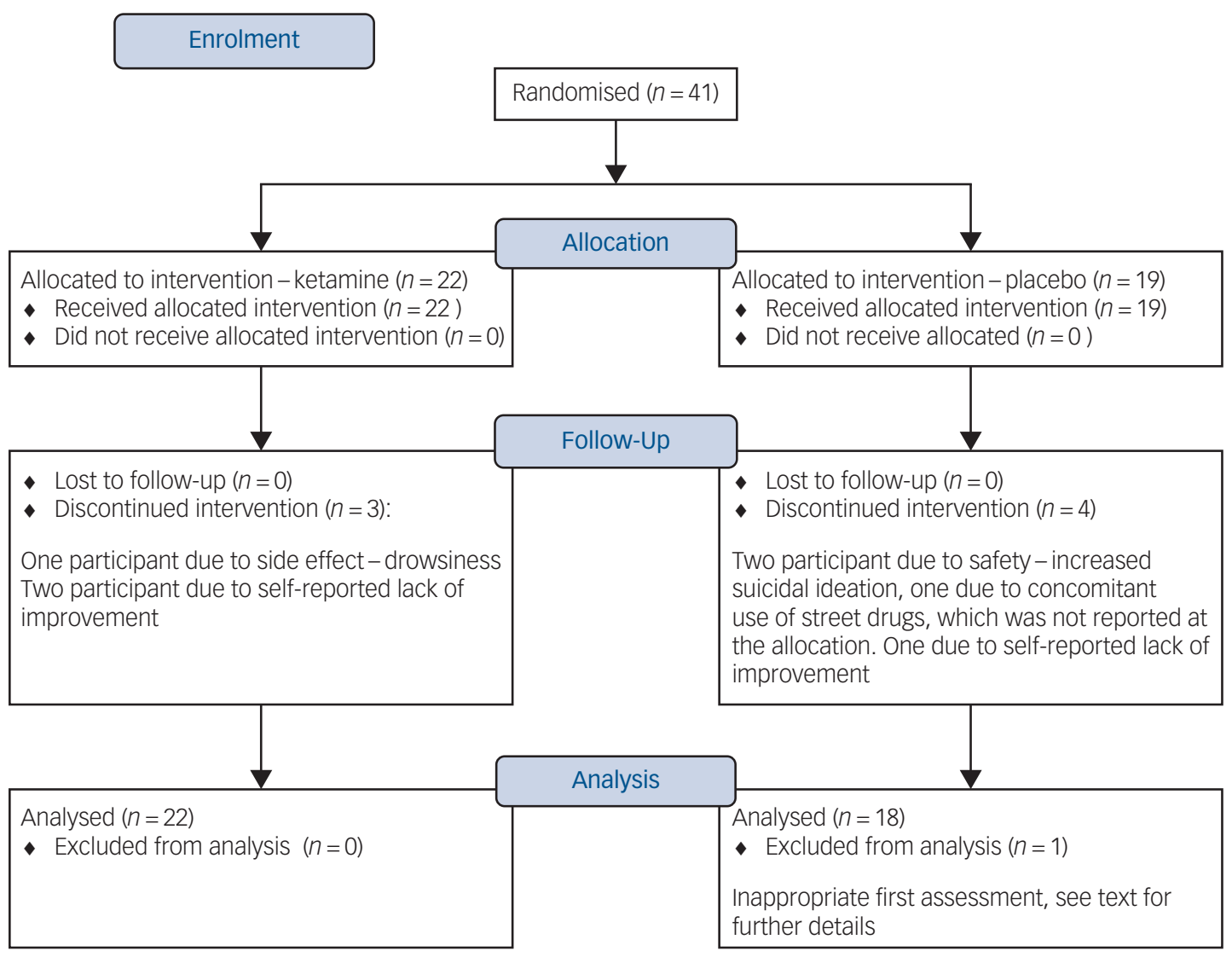

Fig. 1 Study design. 
and prepared respective sealed syringes of active drug or placebo at identical volume, colour and shape for each patient for the duration of the trial. The drug/placebo administration was through oral selfingestion of the syringe content. The participants were masked, and so were their care providers and the research team (excluding the randomising physician).

To reduce interparticipant variability of absorption as much as possible, all sessions were conducted during the morning hours, and patients were asked not to consume food or beverages other than water for the preceding $2 \mathrm{~h}$, as well as coffee or alcohol $12 \mathrm{~h}$ prior to ketamine administration. During the first administration, participants remained under clinical supervision and vital signs monitoring for $6 \mathrm{~h}$ at Tel Aviv Sourasky Medical Center. Blood pressure and heart rate were recorded every $10 \mathrm{~min}$ during the first hour, and then every $30 \mathrm{~min}$ for the following $2 \mathrm{~h}$ and before discharge.

During follow-up, participants were specifically interviewed for side-effects that were recorded in the patient's chart. Following the first administration, if no significant side-effects were observed, participants were given the drug/placebo for home administration. As a result of worries about misuse of ketamine, participants received only the doses required for continued administration until the following meeting (maximum two doses). At each follow-up meeting, participants confirmed their previous drug intakes in order to ensure adherence.

\section{Statistical analyses}

Demographic and clinical characteristics, number of failed pharmacological trials, suicide risk and baseline outcome measures were compared between intervention groups using $t$-test for continuous variables, Mann-Whitney $U$-test for non-parametric variables or $\chi^{2}$-tests for categorical variables.

\section{Sample size determination}

Based on the robust effects of ketamine treatment described in previous literature, the number of individuals per group needed to significantly show an improvement in MADRS scores is relatively small. Based on Singh et al, ${ }^{5}$ for three times weekly treatment and assuming a MADRS improvement of 17.7 points, with a standard deviation of 7.3, with type I error set at 0.05 and aiming at a statistical power of 0.9 , the minimal number of participants per group is $n=5$. For better power, however, we aimed to include at least 18 participants per group.

Study outcomes were analysed using hierarchical linear models (mixed linear model) for continuous outcomes. The intercept was allowed to vary randomly. The analysis was performed in SPSS 25 using maximum likelihood estimation. We examined treatment impact on MADRS controlling for age and gender as covariates, to maximise statistical power and precision of the treatment effect estimate. Ketamine treatment, time of measurement, their interaction and gender were used in the model as fixed effects, and participant and age were entered as random effects. The two time points were compared separately for each treatment using post hoc contrasts, and the resulting probabilities were Bonferroni adjusted. Age was grand-mean centred. Effect sizes were calculated by dividing the adjusted mean difference by the unadjusted pooled standard deviation. Remission and response were analysed using $t$-tests for independent samples.

\section{Number needed to treat (NNT)}

In order to assess the effectiveness of ketamine treatment, the NNT was calculated. NNT represents the number of patients that need to be treated for one of them to benefit compared with a control. It is defined as the inverse of the absolute risk reduction. It was calculated using the formula NNT $=1 /$ CER (control event rate) EER (experimental event rate). Baseline measures and demographic variables were compared between the two groups using $\chi^{2}$ for independence tests, Mann-Whitney $U$-tests or $t$-test for independent samples, depending on the scales of the variables.

\section{Results}

In total 41 participants ( 26 men, 15 women; age range $18-75$ years) were included in the study and randomised to receive ketamine $(n=$ $22)$ or placebo $(n=19)$. Table 1 provides details of demographic and clinical characteristics of study participants.

There was no difference in baseline depression severity between the groups, mean MADRS scores $33.4($ s.d. $=5.5)$ versus 29.99 $($ s.d. $=7.4)(P=0.16)$, for the ketamine and placebo group, respectively. In addition, there were no demographic differences between the two groups at baseline, apart from a female predominance in the placebo group. There were no differences between groups at baseline, in patients' clinical characteristics, apart from more prescribed medications in the ketamine group. A total of 33 (82.5\%) patients

Table 1 Demographic and clinical characteristics of study participants by group

\begin{tabular}{|c|c|c|c|}
\hline Characteristics & $\begin{array}{l}\text { Ketamine } \\
(n=22)\end{array}$ & $\begin{array}{l}\text { Placebo } \\
(n=18)\end{array}$ & $P^{a}$ \\
\hline \multicolumn{4}{|l|}{ Demographic characteristics } \\
\hline Age, years: mean (s.d.) & 38.7 (13.3) & $37.9(13.4)$ & $0.86^{\mathrm{b}}$ \\
\hline Education, years: mean (s.d.) & $14.0(2.2)$ & $13.5(2.5)$ & $0.48^{\mathrm{b}}$ \\
\hline Gender, women: $n$ (\%) & $5(22.7)$ & $10(55.6)$ & $0.03^{\mathrm{C}}$ \\
\hline Unemployed, $n$ (\%) & $10(45.5)$ & $9(50)$ & $0.78^{\mathrm{c}}$ \\
\hline Married or cohabiting, $n$ (\%) & $5(22.7)$ & $5(27.8)$ & $0.71^{\mathrm{C}}$ \\
\hline Israeli native-born, $n$ (\%) & $18(81.8)$ & $15(83.3)$ & $0.90^{C}$ \\
\hline \multicolumn{4}{|l|}{$\begin{array}{l}\text { Blood pressure prior to first } \\
\text { treatment, mean (s.d.) }\end{array}$} \\
\hline Systolic & $122.2(17.1)$ & $129.6(17.9)$ & $0.29^{b}$ \\
\hline Diastolic & $76.9(9.6)$ & $78.1(10.8)$ & $0.31^{\mathrm{b}}$ \\
\hline \multicolumn{4}{|l|}{ Clinical characteristics } \\
\hline $\begin{array}{l}\text { Baseline Montgomery-Åsberg } \\
\text { Depression Rating Scale score, } \\
\text { mean (s.d.) }\end{array}$ & $33.4(5.5)$ & $29.99(7.4)$ & $0.16^{\mathrm{b}}$ \\
\hline $\begin{array}{l}\text { Melancholic features (according to } \\
\text { the } \mathrm{MINI}), n(\%)\end{array}$ & $11(50)$ & $12(66.7)$ & $0.29^{c}$ \\
\hline Personality disorder & 7 (31.8) & $4(22.2)$ & $0.50^{c}$ \\
\hline Anxiety disorder & $13(59.1)$ & $8(44.4)$ & $0.36^{\mathrm{c}}$ \\
\hline Post-traumatic stress disorder & $6(27.3)$ & $4(22.2)$ & $0.71^{\mathrm{C}}$ \\
\hline Obsessive-compulsive disorder & $2(9.1)$ & $2(11.1)$ & $0.83^{\mathrm{c}}$ \\
\hline Panic disorder & $1(4.5)$ & $2(11.1)$ & $0.43^{\mathrm{c}}$ \\
\hline Other psychiatric diagnosis & $4(18.2)$ & $6(33.3)$ & $0.27^{\mathrm{C}}$ \\
\hline $\begin{array}{l}\text { Number of antidepressants in } \\
\text { current episode, } n(\%)\end{array}$ & & & $0.05^{\mathrm{d}}$ \\
\hline 0 & $2(9.1)$ & $4(22.2)$ & \\
\hline 1 & $5(22.7)$ & $5(27.8)$ & \\
\hline 2 & $3(13.6)$ & $3(16.7)$ & \\
\hline 3 & $5(22.7)$ & $6(33.3)$ & \\
\hline 4 & $6(27.3)$ & $0(0)$ & \\
\hline$>5$ & $1(4.5)$ & $0(0)$ & \\
\hline $\begin{array}{l}\text { Suicidal risk (according to the MINI), } \\
\qquad n(\%)\end{array}$ & & & $0.31^{d}$ \\
\hline No risk & $4(19)$ & $1(5.9)$ & \\
\hline Low & $1(4.8)$ & $1(5.9)$ & \\
\hline Intermediate & 7 (33.3) & $5(29.4)$ & \\
\hline High & $9(42.9)$ & $10(58.8)$ & \\
\hline \multicolumn{4}{|c|}{$\begin{array}{l}\text { MINI, Mini International Neuropsychiatric Interview; MADRS, Montgomery-Åsberg } \\
\text { Depression Rating Scale. } \\
\text { a. P-value of the comparison between the two groups. } \\
\text { b. } t \text {-test for independent samples. } \\
\text { c. } \chi^{2} \text { for independence test. } \\
\text { d. Mann-Whitney } U \text {-test. } \\
\text { e. Data are missing for one patient in each group. }\end{array}$} \\
\hline
\end{tabular}


completed the 21-day trial. Three patients dropped out from the ketamine group (13.6\%), two because of reported lack of improvement and one because of complaints of drowsiness. From the placebo group four patients $(22.2 \%)$ dropped out - two were excluded by the research team because of increasing suicidal ideation, one because of concomitant use of street drugs and one because of lack of improvement. One additional participant was retrospectively excluded from the statistical analysis following an internal monitoring process; that participant did not show up for his first assessment. Thus, his assessment was performed via a phone call, and therefore this was incompatible with study requirements.

\section{Main outcome measure: depression}

The linear mixed-model that examined group differences in MADRS score over time showed a statistically significant interaction effect between ketamine and time of measurement $(P=$ 0.045). Bonferroni corrected post hoc analyses showed a statistically significant decrease in MADRS scores in the ketamine group between baseline and all the other time points $(P<0.005)$, including day 21. In the control group a significant decrease was apparent only at $40 \mathrm{~min}$ after initial administration $(P<0.05)$ (Fig. 2). The reduction in the mean MADRAS score at day 21 was 12.75 points for the ketamine group (from 33.4 (s.e. $=2.18$ ) to 20.65 (s.e. $=2.28$ ) versus 2.49 (from $29.99($ s.e. $=2.44)$ to $27.5($ s.e. $=2.61)$ ) points in the placebo group $(P<0.001)$.

\section{Secondary outcomes: response and remission at day 21 - end of trial}

Responders were defined as participants who showed a reduction of at least $50 \%$ from their baseline MADRS scores ${ }^{19}$ on day 21 . As seen in supplementary Fig. 1 (available at https://doi.org/10.1192/bjp.2018. 196) there were significantly more responders in the ketamine group - seven responders $(31.8 \%)$ - compared with one in the placebo group $(5.6 \%)\left(\chi^{2}(1)=4.27, P<0.05\right)$. Remitters were defined as participants who attained MADRS score of $\geq 10$ at day $21 .{ }^{19}$ Six out of 22 participants achieved remission at day 21 in the ketamine group $(27.3 \%)$ as opposed to none in the control group $\left(\chi^{2}(1)=5.78, P<\right.$ $0.05)$, supplementary Fig. 1 . The calculated NNT index for response and remission with ketamine at 21 days were 3.8 and 3.7, respectively. As in our sample there were more women in the placebo group compared with the ketamine group (55.6\% compared with $22.7 \%$ ), gender
Table 2 Side-effects recorded in the ketamine and placebo groups, and their statistical comparison, using $\chi^{2}$ tests for independence

\begin{tabular}{|c|c|c|c|c|c|}
\hline \multirow[b]{2}{*}{ Side-effect } & \multicolumn{2}{|c|}{$\begin{array}{c}\text { Ketamine } \\
(n=22)\end{array}$} & \multicolumn{2}{|c|}{$\begin{array}{c}\text { Placebo } \\
(n=18)\end{array}$} & \multirow[b]{2}{*}{$P\left(\chi^{2}\right)$} \\
\hline & $n$ & $\%$ & $n$ & $\%$ & \\
\hline Euphoria & 4 & 18.2 & 0 & 0.0 & 0.06 \\
\hline Dissociative symptoms & 1 & 4.5 & 0 & 0.0 & 0.36 \\
\hline Dizziness & 4 & 18.2 & 1 & 5.6 & 0.23 \\
\hline Drowsiness & 2 & 9.1 & 1 & 5.6 & 0.67 \\
\hline Nausea & 1 & 4.5 & 2 & 11.1 & 0.43 \\
\hline Vomiting & 0 & 0.0 & 1 & 5.6 & 0.26 \\
\hline Blurred vision & 1 & 4.5 & 0 & 0.0 & 0.36 \\
\hline Headache & 0 & 0.0 & 1 & 5.6 & 0.26 \\
\hline
\end{tabular}

was used as a covariate in the main analysis. However, the effect of gender was not statistically significant.

\section{Safety}

In total, $40 \%$ ( $n=6$, out of 15 with available data) of ketamine participants compared with $18.2 \%(n=2$, out of 11 with available data) in the placebo group $\left(\chi^{2}(1)=1.42, P=0.23\right)$ demonstrated elevation of systolic blood pressure of more than $20 \mathrm{~mm} / \mathrm{Hg}$ at first administration, the peak of this transient elevation was 40 min post-drug administration and it resolved spontaneously within $1 \mathrm{~h}$. Additional immediate common side-effects included euphoria, dizziness and drowsiness, which again resolved in the space of $1 \mathrm{~h}$ and were much milder in later administrations. One participant, however, opted out of the study following a single administration because of mild-to-moderate transient drowsiness. Side-effect profiles are summarised in Table 2. There was an overall trend for more sideeffects in the ketamine group; however, there were no significant differences between the two groups.

\section{Safety follow-up}

An additional evaluation was conducted at day 28 for safety purposes. There was no worsening of depressive symptoms, no 'rebound' effect, and for the ketamine group, at day 28 the effect was maintained with the MADRS scores still significantly lower than baseline.

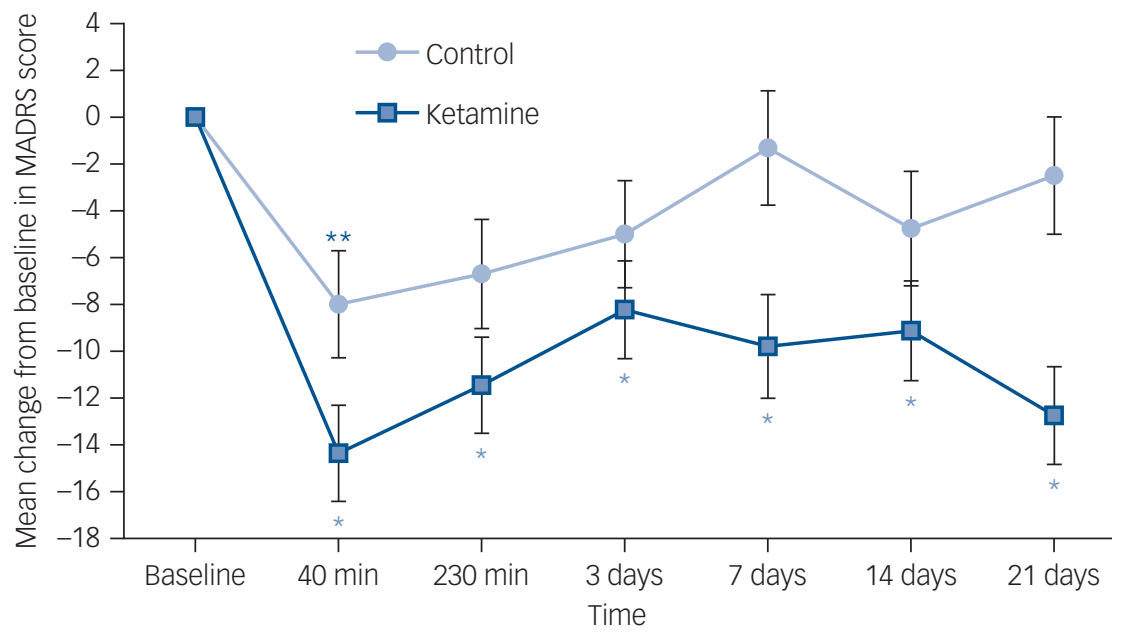

Fig. 2 Change in Montgomery-Åsberg Depression Rating Scale (MADRS) score across time.

Change is from baseline to day 21 in a double-blind study of repeated oral ketamine for out-patients with treatment-resistant depression. ${ }^{*} P<0.005,{ }^{* *} P<0.05$. 


\section{Discussion}

\section{Main findings}

In this study we show, for the first time in a controlled setting, that repeated administration of oral ketamine can lead to amelioration of depressive symptoms in patients with TRD in the community, and may be well tolerated. Crucially, our results indicate that oral ketamine can induce remission in a significant proportion of patients previously defined as having TRD.

Current antidepressant pharmacotherapy fails to achieve remission in about $30 \%$ of patients, even when combining multiple antidepressants and applying various augmentation strategies. ${ }^{1}$ This substantial portion of patients who fail to achieve remission are considered to have $\mathrm{TRD}^{2}$ and are at least twice as likely to be admitted to hospital and require more out-patient visits. ${ }^{20}$ TRD is therefore a costly disease, associated with extensive use of depression-related and general medical services. ${ }^{21}$ In addition, TRD is related to higher suicide risk. ${ }^{22}$ Resistance to antidepressive treatment has many aspects and an inconsistent definition. ${ }^{23}$ Although it can fit a more complex model ${ }^{24}$ and include other biological treatments and psychotherapy, in this study we used the widespread definition of TRD as failure to achieve remission despite the use of at least two different antidepressants at adequate dose and duration. ${ }^{2}$

\section{Route of administration of ketamine}

Intravenous ketamine has been repeatedly shown to ameliorate depressive symptoms in previously studies of patients with TRD.,4 Intravenous infusion, however, severely limits the applicability in out-patient settings. In this respect, the recent interest in intranasal administration may offer a new method for treating out-patients with ketamine ${ }^{6}$ or esketamine. ${ }^{25}$ This novel route of administration, however, has yet to be studied for at-home usage. Oral at-home administration of ketamine, on the other hand, has been well described in patients with chronic pain. ${ }^{9}$ Accordingly, there is a pressing interest in exploring and advancing the oral administration of ketamine in individuals with $\mathrm{TRD},{ }^{14}$ which would represent a preferable option, because of higher clinically applicability, lower costs and higher convenience for patients. This route of administration, however, has scarcely been reported in patients with psychiatric conditions, probably because of low and variable bioavailability. ${ }^{9}$ The current study, therefore, presents a novel translation into psychiatric practice.

\section{Comparison with findings from other studies}

Notwithstanding the limitations of comparing different study results, our results are comparable with others who studied different routes of administration. Singh et $a l^{5}$ studied repeated ketamine infusions and reported up to 18 MADRS points reduction at day 15 , whereas we found a reduction of 12.75 points in the MADRS score at day 21. Our result are also comparable with those described for intranasal administration of esketamine, $\mathrm{S}$ enantiomer of ketamine. Daly et $a l^{25}$ reported treatment effects of up to 12.5 MADRS points reduction relative to placebo. In our study this difference was 10.26 (12.75 reduction in the ketamine group v. 2.49 reduction in the placebo group). In contrast, $\mathrm{Al}$ Shirawi et $a l,{ }^{13}$ in their series of case report of oral ketamine for TRD reported disappointing results. However, they have used a relatively ultralow non-adjusted dose of $50 \mathrm{mg}$, compared with the $1 \mathrm{mg} / \mathrm{kg}$ dose that we used and that proved to be effective and safe. Furthermore, we observed a cumulative effect of the treatment, with the best clinical outcome obtained at the end of the study (day 21). One possibility is that, metabolites produced by gastrointestinal absorption may affect the pharmacodynamic and pharmacokinetic properties of the immediate antidepressant proteogenetic process, but still induce relevant therapeutic plastic synaptic changes with longer treatment duration. This speculation, of course, is yet to be explored. Notably, 1 week after treatment cessation, the treatment effect was sustained.

\section{Response, remission and adherence to treatment}

Key clinical outcomes in assessing new approaches to the treatment of TRD are response and remission. In the current study, a significant proportion of patients who were unresponsive by definition to several other lines of antidepressants treatment attained clinical remission. This finding cannot be overstated. The calculated NNT for attaining clinical remission with add-on ketamine in individuals with TRD was 3.7, whereas no patient went into remission within the placebo group. Moreover, the ketamine group was treated with significantly more medications at the time of enrolment, but still with no clinical beneficial effect. This serves to further demonstrate the fact that these patients had not benefited from a multitude of existing drug treatments.

Adherence to treatment is a chief concern in psychiatry in general and in TRD in particular. Adherence may reflect therapeutic efficacy but also the ability to tolerate side-effects. A total of $86.3 \%$ of the ketamine group completed the study compared with $77.8 \%$ of the control group, which might reflect adherence to treatment. Overall, ketamine was well tolerated and the most common side-effects in patients were mild dizziness and euphoria, both resolving within the space of an hour, and were much milder in later administrations.

\section{Real life study design}

Concomitant psychiatric diagnosis and personality disorders are more prevalent in TRD than in other depressive disorders. ${ }^{2,20}$ Thus, this study was designed to be more of a 'real-life study' in the sense that we did not exclude participants who had certain concomitant psychiatric disorders. One of every four participants had concomitant post-traumatic stress disorder, more than half had anxiety disorders and more than a quarter struggled with personality disorders. Although this approach raises methodological challenges, it captures the complexities of real-life clinical psychiatry.

\section{Obstacles to ketamine treatment}

There are several obstacles to out-patient treatment with ketamine. First, ketamine is a potent psychotomimetic agent, and should be used with caution in neuropsychiatric diseases. This is especially true when employing this medication in the out-patient setting. Second, the safety of ketamine for patients with psychotic symptoms has not been well established. Thus, as a precaution, we excluded individuals with previous psychotic symptoms. Third, evidence for determining optimal dosing and regimen are limited. As a preliminary approach, our choice of $1 \mathrm{mg} / \mathrm{kg}$ thrice weekly seems to have had the desirable efficacy with marginal side-effects. The optimal dosing regimens remain to be explored.

\section{Limitations}

Efficient masking was one of the major limitations in performing this study. This is an inherent problem in all ketamine studies because of ketamine's rapid psychomimetic effect. To this end, some studies used an active placebo such as midazolam. ${ }^{4,26}$ Those studies, however, have used a single dose of active placebo, whereas our study design required repeated placebo self-administrations in the home setting. Thus, using any psychoactive drug, especially with addiction potential, might have compromised our participant's health. Two other potential obstacles for masking are ketamine's bitter taste and the cardiovascular effect. In order to 
deal with the problem of the bitter taste in terms of masking the raters were not present in the room at the time of drug administration. The participants where not informed of a taste difference, and we have no reason to assume that they knew of such a difference. The third obstacle for masking was the cardiovascular effect, which can be apparent to raters and participants alike. We, however, found no statistically significant difference in pulse nor in blood pressure between the two groups. In conclusion, flavouring the placebo and using different efficacy and safety raters is advised in future studies to maintain proper masking. Because of the low and variable bioavailability of oral ketamine, blood levels should be taken into account when considering the antidepressant effect of oral ketamine, ${ }^{14}$ lack of blood levels is another limitation of this study, and future studies should address this issue.

\section{Implications}

To conclude, this proof-of-concept study suggests that repeated oral ketamine could provide a safe, feasible and effective intervention for patients with TRD in a community setting. Our results, although promising, cannot yet be applied to clinical practice without larger, randomised studies. Such studies are needed to address questions such as optimal dosing regimens, patient selection and treatment duration to properly assess the safety of long-term ketamine usage, the risk of misuse and the restricted means appropriate for at-home prescription. Furthermore, a longer follow-up phase should be considered. Finally, it seems plausible to examine the possible role of oral ketamine in the treatment of acute suicidal ideation as well as additional psychopathologies.

\footnotetext{
Yoav Domany, MD (D), Senior Psychiatrist, Department of Psychiatry, Tel Aviv Sourasky Medical Center and Sackler School of Medicine, Tel Aviv University, Israel and

Postdoctoral Research Fellow, Department of Psychiatry and Behavioral Neuroscience

University of

cher, Sagol Brain

Institute Tel-Aviv Sourasky Medical Center and Clinical Manager, Brainsway Ltd, Israel;

Ricardo Tarrasch, PhD, Psychologist, Researcher, Statistician and Educator, Assistant

Professor, School of Education and School of Neuroscience, Tel Aviv University, Israel;

Roi Meidan, MD, Physician for Emergency Medicine, Department of Emergency

Medicine, Tel Aviv Sourasky Medical Centre, Israel Olga Litvak-Lazar, BA, Research

Coordinator, Department of Psychiatry, Tel Aviv Sourasky Medical Centre, Israel;

Nadav Stoppleman, PhD, Researcher, Center for Brain Functions Wohl Institute for

Advanced Imaging, Tel-Aviv Sourasky Medical, Israel; Shaul Schreiber, MD, Director,

Department of Psychiatry, Tel Aviv Sourasky Medical Center and Professor of Psychiatry,

Tel Aviv University, Sackler Faculty of Medicine and Member, Sagol School of

Neuroscience, Tel Aviv University, Israel; Miki Bloch, MD, Head of Inpatient Psychiatric

Ward, Tel Aviv Sourasky Medical Centre and Sackler School of Medicine, Tel Aviv

University, Israel; Talma Hendler, MD, PhD, Head of Sagol Brain Institute, Wohl Institute

University, Isral; Talma Hendler, $M$, Pr

Sagol School of Neuroscience, Tel Aviv University, Israel; Haggai Sharon, MD, Senior

Physician, Institute of Pain Medicine, Department of Anesthesiology, Pain and Critical

Care Medicine, Tel Aviv Sourasky Medical Centre and Researcher, Center for Brain

Functions, Wohl Institute for Advanced Imaging, Tel-Aviv Sourasky Medical Centre and

Sackler School of Medicine, Tel Aviv University, Israel and Pain Management \&

Neuromodulation Centre, Guy's \& St Thomas' NHS Foundation Trust, UK

Correspondence: Yoav Domany, 5546 East Galbraith Road, Cincinnati, Ohio 45236, USA Email: yoavdomany@gmail.com

First received 22 May 2018, final revision 8 Aug 2018, accepted 16 Aug 2018
}

\section{Funding}

This work was supported by the Tel Aviv Medical Center Brain Grant 2016 (Y.D and H.S) and the NARSAD Young Investigator Award (H.S) provided by the Brain\&Behavior Research Foundation and the Oxley Foundation.

\section{Acknowledgements}

We would like to thank our participants and their families. In addition, we thank Avi Lougass Keren Armoni-Domany, Yana Beitelman, Sharona Even-Tov, Noam Goldway, Libat Weizman, Nilli Green, and Cheryl B. McCullumsmith for their help and support.

\section{Supplementary material}

Supplementary material is available online at https://doi.org/10.1192/bjp.2018.196.

\section{References}

1 Rush AJ, Trivedi MH, Wisniewski SR, Nierenberg AA, Stewart JW, Warden D, et al. Acute and longer-term outcomes in depressed outpatients requiring one or several treatment steps: a STAR*D report. Am J Psychiatry 2006; 163 1905-17.

2 Fava M. Diagnosis and definition of treatment-resistant depression. Biol Psychiatry 2003; 53: 649-59.

3 Zarate Jr CA, Singh JB, Carlson PJ, Brutsche NE, Ameli R, Luckenbaugh DA, et al. A randomized trial of an $\mathrm{N}$-methyl-D-aspartate antagonist in treatmentresistant major depression. Arch Gen Psychiatry 2006; 63: 856-64.

4 Murrough JW, Iosifescu DV, Chang LC, Al Jurdi RK, Green CE, Perez AM, et al. Antidepressant efficacy of ketamine in treatment-resistant major depression: a two-site randomized controlled trial. Am J Psychiatry 2013; 170: $1134-42$.

5 Singh JB, Fedgchin M, Daly EJ, De Boer P, Cooper K, Lim P, et al. A double-blind, randomized, placebo-controlled, dose-frequency study of intravenous ketamine in patients with treatment-resistant depression. Am J Psychiatry 2016; 173: 816-26.

6 Lapidus KA, Levitch CF, Perez AM, Brallier JW, Parides MK, Soleimani L, et al. A randomized controlled trial of intranasal ketamine in major depressive disorder. Biol Psychiatry 2014; 76: 970-6.

7 Lara DR, Bisol LW, Munari LR. Antidepressant, mood stabilizing and procognitive effects of very low dose sublingual ketamine in refractory unipolar and bipolar depression. Int J Neuropsychopharmacol 2013; 16: 2111-7.

8 Galvez V, O'Keefe E, Cotiga L, Leyden J, Harper S, Glue P, et al. Long-lasting effects of a single subcutaneous dose of ketamine for treating melancholic depression: a case report. Biol Psychiatry 2014; 76: e1-2.

9 Peltoniemi MA, Hagelberg NM, Olkkola KT, Saari TI. Ketamine: a review of clinical pharmacokinetics and pharmacodynamics in anesthesia and pain therapy. Clin Pharmacokinet 2016; 55: 1059-77.

10 Iglewicz A, Morrison K, Nelesen RA, Zhan T, Iglewicz B, Fairman N, et al Ketamine for the treatment of depression in patients receiving hospice care: a retrospective medical record review of thirty-one cases. Psychosomatics 2015; 56: 329-37.

11 Jafarinia M, Afarideh M, Tafakhori A, Arbabi M, Ghajar A, Noorbala AA, et al. Efficacy and safety of oral ketamine versus diclofenac to alleviate mild to moderate depression in chronic pain patients: a double-blind, randomized, controlled trial. J Affect Disord 2016; 204: 1-8.

12 Swiatek KM, Jordan K, Coffman J. New use for an old drug: oral ketamine for treatment-resistant depression. BMJ Case Rep 2016; 2016: 216088.

13 Al Shirawi MI, Kennedy SH, Ho KT, Byrne R, Downar J. Oral ketamine in treatment-resistant depression: a clinical effectiveness case series. J Clin Psychopharmacol 2017; 37: 464-7.

14 Schoevers RA, Chaves TV, Balukova SM, Rot MA, Kortekaas R. Oral ketamine for the treatment of pain and treatment-resistant depression. Br J Psychiatry 2016; 208: 108-13.

15 Lecrubier Y, Sheehan DV, Weiller E, Amorim P, Bonora I, Sheehan K, et al. The Mini International Neuropsychiatric Interview (MINI). A short diagnostic structured interview: reliability and validity according to the CIDI. Eur Psychiatry 1997; 5: 224-31.

16 Montgomery SA, Asberg M. A new depression scale designed to be sensitive to change. Br J Psychiatry 1979; 134: 382-9.

17 Zimmerman M, Posternak MA, Chelminski I. Defining remission on the Montgomery-Asberg depression rating scale. J Clin Psychiatry 2004; 65: 163-8.

18 Murrough JW, Perez AM, Pillemer S, Stern J, Parides MK, aan het Rot M, et al. Rapid and longer-term antidepressant effects of repeated ketamine infusions in treatment-resistant major depression. Biol Psychiatry 2013; 74: $250-6$

19 Zimmerman M, Posternak MA, Chelminski I. Derivation of a definition of remission on the Montgomery-Asberg depression rating scale corresponding to the definition of remission on the Hamilton rating scale for depression. J PSychiatr Res 2004; 38: 577-82.

20 Crown WH, Finkelstein S, Berndt ER, Ling D, Poret AW, Rush AJ, et al. The impact of treatment-resistant depression on health care utilization and costs. J Clin Psychiatry 2002; 63: 963-71.

21 Mrazek DA, Hornberger JC, Altar CA, Degtiar I. A review of the clinical, economic, and societal burden of treatment-resistant depression: 1996-2013. Psychiatr Serv 2014; 65: 977-87. 
22 Kornstein SG, Schneider RK. Clinical features of treatment-resistant depression. J Clin Psychiatry 2001; 62 (suppl 16): 18-25.

23 McIntyre RS, Filteau MJ, Martin L, Patry S, Carvalho A, Cha DS, et al. Treatmentresistant depression: definitions, review of the evidence, and algorithmic approach. J Affect Disord 2014; 156: 1-7.

24 Ruhe HG, van Rooijen G, Spijker J, Peeters FP, Schene AH. Staging methods for treatment resistant depression. A systematic review. J Affect Disord 2012; 137 35-45.

25 Daly EJ, Singh JB, Fedgchin M, Cooper K, Lim P, Shelton RC, et al. Efficacy and safety of intranasal esketamine adjunctive to oral antidepressant therapy in treatment-resistant depression: a randomized clinical trial. JAMA Psychiatry 2018; 75: 139-48.

26 Grunebaum MF, Galfalvy HC, Choo TH, Keilp JG, Moitra VK, Parris MS, et al. Ketamine for rapid reduction of suicidal thoughts in major depression: a midazolam-controlled randomized clinical trial. Am J Psychiatry 2017: 175: 327-35. 